\title{
Search for charmed hadrons in the OPERA experiment
}

\author{
A. Pastore ${ }^{1, a}$ on behalf of the OPERA Collaboration \\ ${ }^{1}$ INFN Sezione di Bari, I-70126 Bari, Italy
}

\begin{abstract}
The OPERA experiment was designed to study muon neutrino to tau neutrino oscillations in appearance mode, using the CERN to Gran Sasso (CNGS) high energy neutrino beam $730 \mathrm{~km}$ far from the source. From 2008 to 2012, CNGS neutrinos interactions were recorded in the OPERA detector, which includes target units made of lead plates alternated with emulsion films and electronic tracker planes. The on-going analysis is aimed at the detection of short-lived particle decays occurring over distances of the order of $1 \mathrm{~mm}$ from the neutrino interaction point. It has allowed identifying charmed hadrons together with the tau lepton decay candidates that have established $v_{\mu} \rightarrow v_{\tau}$ oscillations with a significance of $4.2 \sigma$. The procedure applied in OPERA to detect short-lived particle decays and its application to the search for charmed hadrons will be discussed here in detail.
\end{abstract}

\section{Introduction}

The Oscillation Project with Emulsion tRacking Apparatus (OPERA) [1] was designed to directly observe tau-neutrino appearance in the CERN Neutrinos to Gran Sasso (CNGS) [2] high energy muonneutrino beam.

The CNGS beam was produced at CERN and delivered at the Gran Sasso Underground Laboratory of INFN (LNGS), $730 \mathrm{~km}$ away, where the OPERA detector is located. It ran for five years and completed its operation in December 2012 having delivered an integrated intensity of about $18 \times 10^{19}$ protons on target.

So far, OPERA has observed four $v_{\tau}$ Charged Current (CC) interaction candidate events [4] [5] [6] [7], consistent with the expectation of the standard oscillation framework at the atmospheric scale. This finding, representing the first direct evidence of $v_{\mu} \rightarrow v_{\tau}$ oscillations in appearance mode, as well as other physics results of the experiment are discussed in detail in [8] [9]. The on-going oscillation analysis is aimed at the detection of the decays of the short-lived $\tau$ lepton originating from the oscillated $v_{\tau}$ CC interactions which occur over distances of the order of $1 \mathrm{~mm}$ from the neutrino interaction point at CNGS energies.

Nuclear emulsions, instrumenting the OPERA target, are tracking detectors particularly well suited for the study of these decay processes: using the complementary information provided by electronic detectors, their event topology and kinematics can be fully reconstructed.

In the following, a short description of the OPERA detector and event analysis is given, the procedure applied to detect short-lived particle decays is discussed in detail together with its application to the

\footnotetext{
a e-mail: alessandra.pastore@ba.infn.it
} 
search for charmed hadrons in the full data sample of CC events collected in 2008 and 2009 runs as well as in a subsample of the statistics collected in the 2010 run. Charmed hadrons have indeed masses and lifetimes similar to those of the $\tau$ lepton and their detection is a powerful tool in order to validate the procedure for the detection of $v_{\tau}$ appearance.

\section{OPERA detector and event analysis}

The OPERA hybrid apparatus consists of two identical Super Modules as shown in figure 1. Each

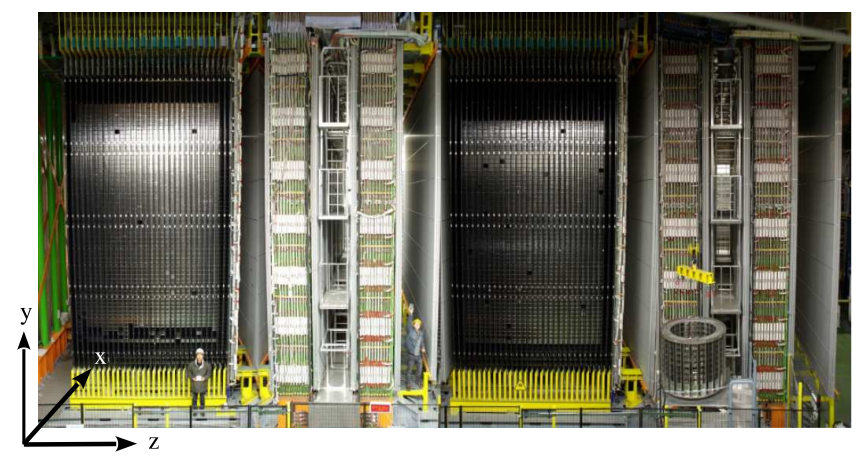

Figure 1. View of the OPERA detector

Super Module is made up of a target area followed by a muon spectrometer. A single target section is arranged in vertical "walls", across the beam direction.

Every wall is filled with target elementary constituents, the so-called lead/emulsion "bricks" and all are followed by two planes of plastic scintillator strips, one per transverse direction, called Target Trackers (TT). The TT were designed to locate, on a centimetric scale, the brick where neutrino interactions occured.

The lead-emulsion structure of the brick makes possible the reconstruction of neutrino interactions and short-lived particles decays with a sub-micron accuracy. It also allows charged particles momenta evaluation by Multiple Coulomb Scattering, $\mu / \pi$ identification by $d E / d x$ measurements and electromagnetic shower reconstruction. It consists of 57 nuclear emulsion films, interleaved with $1 \mathrm{~mm}$ thick lead plates, with a total volume of $7.9 \times 10.2 \times 12.8 \mathrm{~cm}^{3}$ and mass of $8.3 \mathrm{~kg}$. OPERA emulsion films are made of two $45 \mu \mathrm{m}$-thick sensitive layers deposited on each side of a $205 \mu \mathrm{m}$ plastic base.

An additional tightly packed doublet of emulsion films (Changeable Sheets, CS) is glued to the downstream face of the brick, in order to validate the brick selection and connect the electronic detectors predictions to the brick.

The overall target mass is about $1.2 \mathrm{kt}$. Each spectrometer consists of a dipolar magnet instrumented with planes of Resistive Plate Chambers (Inner Tracker) and drift tubes (Precision Tracker) detectors in order to identify muons mainly coming from $v_{\mu} \mathrm{CC}$ interactions and measure their momentum and charge. The charge-sign misidentification probability was estimated to be about $0.3 \%$ up to $50 \mathrm{GeV} / \mathrm{c}$; the momentum resolution is about $20 \%$ in the same kinematical range. A veto system, consisting of planes of glass Resistive Plate Chambers, is placed in front of the first Super Module in order to tag the interactions occurring in the upstream rock.

The apparatus is equipped with an automatic machine (Brick Manipulator System, BMS), allowing the online removal of bricks from the detector, together with some ancillary facilities devoted to the 
emulsion films' handling. Specially designed and dedicated European and Japanese scanning stations perform the nuclear emulsion films measurement. The experimental apparatus and measurement techniques are described in more details in [3].

The events triggered by the neutrino beam, in coincidence with the two $10.5 \mu$ s long CNGS spills $50 \mathrm{~ms}$ distant ("on-time" events), are those used for neutrino oscillation studies. Every time a charged particle belonging to an "on-time" event produces a signal in the TT, a brick finding algorithm selects the bricks with the highest probability to contain the neutrino interaction. The efficiency of this procedure is $83 \%$ when up to 4 bricks per event are processed.

The selected brick is removed from the target by the BMS and the corresponding CS doublet is detached from it and developed in a dedicated underground facility. The two emulsion films are scanned in one of the dedicated scanning stations of the Collaboration. The measurement of emulsion films is performed through fast automated microscopes with a scanning speed greater than $20 \mathrm{~cm}^{2} / \mathrm{h}$. The tracking efficiency was evaluated to be about $90 \%$, the position resolution being at the sub-micron level. The angular resolution is of the order of one milliradian. The residuals between electronic detectors' predictions and CS tracks are $\sim 1 \mathrm{~cm}$.

If any track originating from the interaction is detected in the CS, the brick is brought to the surface laboratory and exposed to high-energy cosmic rays for alignment purposes and then unpacked. Its emulsion films are developed and sent to the scanning laboratories of the Collaboration to find the neutrino interaction location within the brick and from it search for decays of short-lived secondary particles.

All CS tracks are searched for in the most downstream film of the brick. The CS to brick connection is achieved with a position accuracy smaller than $100 \mu \mathrm{m}$ and a slope accuracy of the order of 10 mrad. Tracks that have been successfully located in a CS doublet are followed upstream through the corresponding brick (scan-back) until they stop. This is interpreted as a signature of either a primary or a secondary vertex.

A $2 \mathrm{~cm}^{3}$ volume is scanned around the stopping points in order to reconstruct the vertex topology with micrometric precision.

In order to detect decay topologies, every located vertex is carefully investigated by means of a dedicated procedure [10] which will be discussed in detail in the following.

\section{Search for short-lived particle decays}

Tau decays inside a brick may be classified in two categories depending on the $\tau$ decay length. A decay is defined as short if it occurs in the same lead plate as the $v_{\tau} \mathrm{CC}$ interaction or in the first 45 mm-thick emulsion layer downstream of it. Otherwise, the decay is defined as long.

In the first case, daughter tracks have a large impact parameter with respect to the neutrino vertex while, in case of long decays, they typically appear as tracks (extra-tracks) not attached to the reconstructed neutrino interaction point, starting in the emulsion film immediately downstream of it, or even farther. Depending on the topology under study, a dedicated decay search procedure is applied. Its main steps, sketched in figure 2, are described below.

\subsection{The extra-track and in-track searches}

Once the neutrino interaction point is located in the measured volume, additional extra-tracks are searched for therein with the aim to assess the event topology. A track is selected if it crosses at least three emulsion films and shows an impact parameter with respect to the vertex smaller than $300 \mu \mathrm{m}$ $(500 \mu \mathrm{m})$, depending on whether the longitudinal distance $\Delta \mathrm{z}$ of the most upstream segment of the 


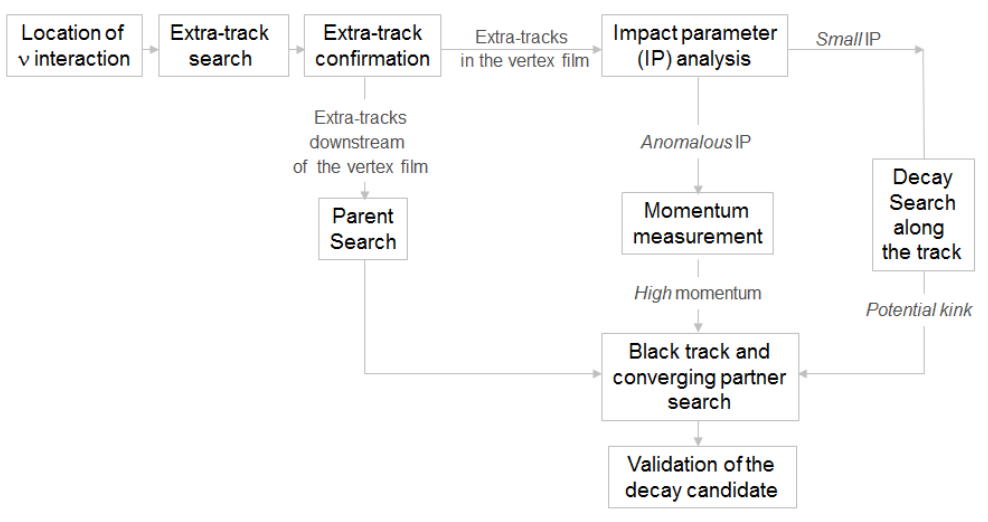

Figure 2. Main steps of the OPERA decay search procedure

track with respect to the vertex is smaller (larger) than $1 \mathrm{~mm}$. To enhance the signal to noise ratio, a cut requiring $\Delta \mathrm{z} \leq 3.6 \mathrm{~mm}$ is also applied. In case of Neutral Current (NC) events the search for extra-tracks is extended to two emulsion films upstream of the vertex and a maximum impact parameter of $500 \mu \mathrm{m}$ between the track end the vertex is accepted. The nature of each selected track is then assessed by visual inspection.

Genuine extra-tracks starting downstream of the vertex film are further analysed as possible $\tau$ decay daughters. Any candidate parent track showing an impact parameter with respect to the reconstructed vertex smaller than $10 \mu \mathrm{m}$ and a minimum distance from the possible daughter track smaller than 20 $\mu \mathrm{m}$ is then validated by visual inspection.

Genuine extra-tracks starting in the vertex film, together with primary tracks, are analysed on the basis of their impact parameter to detect possible short decay topologies. If a track is found to have an impact parameter larger than $10 \mu \mathrm{m}$ for $\Delta \mathrm{z} \leq 500 \mu \mathrm{m}$ or larger than $(5+0.01 \times \Delta \mathrm{z}) \mu \mathrm{m}$ for $\Delta \mathrm{z}>$ $500 \mu \mathrm{m}$, its momentum is then estimated in order to assess whether the observed anomalous value can be explained in terms of scattering in the traversed lead thickness or still remains a hint of a decay.

Searches for heavily ionising particles [11] and for possible converging patterns complement the topological definition of the primary neutrino interaction inside the OPERA brick and take profit of the improvements in wide-angle track acceptance recently obtained with the automatic nuclear emulsion scanning systems in use in the OPERA experiment.

In order to enhance the sensitivity to 1-prong short decay topologies, possible kinks along the event-related tracks are searched for measuring the largest angular difference in its 4 most upstream segments and comparing this value to the RMS of the angular deviations along the track (in-track search). The track is further analysed if this ratio is larger than 5 and the kink angle is greater than $0.015 \mathrm{rad}$.

\subsection{Validation of the decay topologies}

Whenever the decay search procedure identifies an event topology compatible with a short-lived particle decay, a full kinematical analysis of the event is performed in order to confirm it as a signal event for OPERA, a $v_{\mu} \mathrm{CC}$ interaction with the production of a charmed hadron (and its consequent decay) or a background event. The measurements and the discriminating variables used in this analysis are described in detail in [5]. 
Table 1. OPERA decay search efficiency for different charmed hadrons

\begin{tabular}{cccc}
\hline & \multicolumn{3}{c}{ Decay search efficiency } \\
\cline { 2 - 4 } Particle & Short topology & Long topology & Combined \\
\hline $\mathrm{D}^{0}$ & $0.22 \pm 0.02$ & $0.65 \pm 0.05$ & $0.39 \pm 0.04$ \\
\hline $\mathrm{D}^{+}$ & $0.20 \pm 0.05$ & $0.41 \pm 0.09$ & $0.28 \pm 0.05$ \\
\hline $\mathrm{D}_{\mathrm{s}}^{+}$ & $0.18 \pm 0.09$ & $0.56 \pm 0.30$ & $0.33 \pm 0.13$ \\
\hline$\Lambda_{\mathrm{c}}^{+}$ & $0.10 \pm 0.02$ & $0.62 \pm 0.16$ & $0.31 \pm 0.07$ \\
\hline
\end{tabular}

\section{Charmed hadrons search}

Charmed hadrons represent one of the main background sources for the OPERA experiment. Their masses and lifetimes are indeed similar to those of the $\tau$ lepton and the detection of their production and decay represents an important tool to validate the procedure described in Sect. 3.

In the search for charm production in OPERA, this procedure is applied to the full data sample of 2008 and 2009 runs and to a subsample of 2010 data (all NC events and CC events with a reconstructed negative muon with $p_{\mu}<15 \mathrm{GeV}$ ) for a total of $2925 v_{\mu}$ CC events located inside an OPERA brick. Figure 3 shows a $v_{\mu} \mathrm{CC}$ interaction reconstructed in the 2008 data sample, with a candidate $\mathrm{D}^{0}$ particle decaying in 4 prongs. In total, 50 decay candidate events are observed in the analysed data sample.

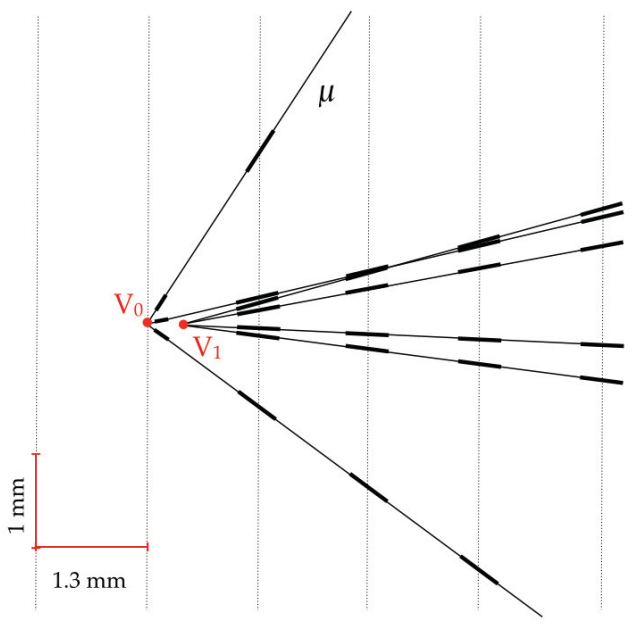

Figure 3. A reconstructed $v_{\mu} \mathrm{CC}$ interaction with a candidate charmed hadron in the final state

The expected charm yield is calculated in the OPERA simulation framework. Taking into account the latest result of the CHORUS experiment on charm production cross-sections [12], at the CNGS energies the total charm production rate relative to the $\mathrm{CC}$ interaction cross-section is evaluated to be $\sigma\left(v_{\mu} N \rightarrow \mu C X\right) / \sigma\left(v_{\mu} N \rightarrow \mu X\right)=(4.49 \pm 0.26) \%$. The results of the study of the decay search efficiency for different charmed hadrons is summarised in table 1.

Hadronic re-interactions constitute the main source of background to charm decay events while a small contribution (about 13\% of the total) comes from the decays of strange particles. As shown in detail in table 2, $(40 \pm 3)$ charm and $(14 \pm 3)$ background events are expected in the analysed sample. Figures 4 and 5 show the distribution of relevant variables for both data and simulation. The 
Table 2. Summary of expected charm and background events compared to observed events

\begin{tabular}{lrrrr}
\hline \multirow{2}{*}{ Decay topology } & \multicolumn{4}{c}{ Events } \\
\cline { 2 - 5 } & $\begin{array}{c}\text { Expected } \\
\text { charm }\end{array}$ & $\begin{array}{c}\text { Expected } \\
\text { background }\end{array}$ & $\begin{array}{c}\text { Expected } \\
\text { total }\end{array}$ & Observed \\
\hline 1-prong & $21 \pm 2$ & $9 \pm 3$ & $30 \pm 4$ & 19 \\
\hline 2-prong & $14 \pm 1$ & $4 \pm 1$ & $18 \pm 2$ & 22 \\
\hline 3-prong & $4 \pm 1$ & $1.0 \pm 0.3$ & $5 \pm 1$ & 5 \\
\hline 4-prong & $0.9 \pm 0.2$ & - & $0.9 \pm 0.2$ & 4 \\
\hline Total & $40 \pm 3$ & $14 \pm 3$ & $54 \pm 4$ & 50 \\
\hline
\end{tabular}

Kolmogorov-Smirnov test is applied and indicates that data and Monte Carlo are in reasonable agreement. The results reported in table 2, together with the shape comparisons illustrated in figures 4 and 5 , show a satisfactory agreement between observed and simulated charm data and, as a consequence, they also validate the method chosen to detect $v_{\tau}$ appearance and confirm that its efficiency is well understood.

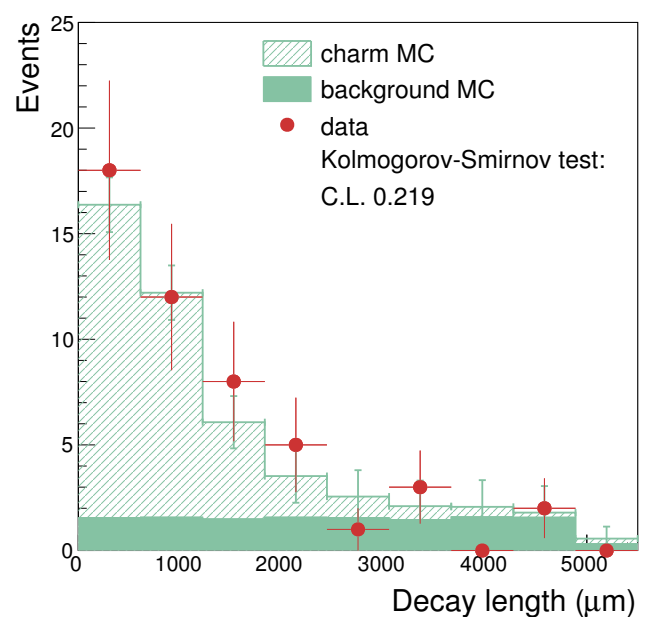

Figure 4. Comparison between observed $v_{\mu} \mathrm{CC}$ interactions with candidate charm decays and MC expectations: decay length distribution of the candidate charmed particles

\section{Conclusions}

The direct observation of short-lived particle decays is a crucial task in the OPERA neutrino oscillation experiment. A dedicated procedure, named decay search, is systematically applied to each neutrino interaction located in a target brick to search for oscillated $v_{\tau} \mathrm{CC}$ candidates. This procedure also allows the detection of $v_{\mu} \mathrm{CC}$ interactions with charmed hadrons in the final state. Its application to a subsample of OPERA data from 2008, 2009 and 2010 runs led to detect 50 charm decay candidate events while $54 \pm 4$ charmed particle events were expected. The comparison between observed and 


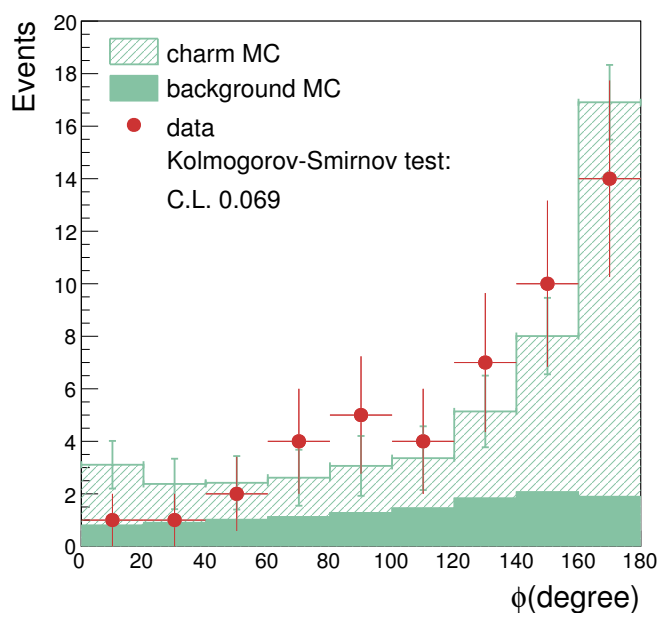

Figure 5. Comparison between observed $v_{\mu} \mathrm{CC}$ interactions with candidate charm decays and MC expectations: distribution of the angle between the candidate charmed hadron and the primary muon in the $v$ transverse plane

simulated data for several relevant variables has shown to be satisfactory, too. Furthermore these results validate the methods used to detect $v_{\tau}$ appearance in the OPERA experiment.

\section{References}

[1] M. Guler et al. [OPERA Collaboration], Experimental Proposal, CERN 98-02, INFN/AE98/05 (1998); M. Guler et al. [OPERA Collaboration], Status Report on the OPERA experiment, CERN/SPSC 2001-025, LNGS-EXP 30/2001 add. 1/01

[2] G. Acquispace et al., CERN-98-02 (1998); R. Bailey et al., CERN-SL/99-034 (1999); A. E. Ball et al., CERNSL/ Note-2000-063 (2000)

[3] R. Acquafredda et al. [OPERA Collaboration], JINST 04,P04018 (2009)

[4] N. Agafonova et al. [OPERA Collaboration], Phys. Lett. B 691, 138-145 (2010)

[5] N. Agafonova et al. [OPERA Collaboration], JHEP 11, 036 (2013)

[6] N. Agafonova et al. [OPERA Collaboration], Phys. Rev. D 89, 051102(R) (2014)

[7] N. Agafonova et al. [OPERA Collaboration], to appear on Prog. Theor. Exp. Phys. 258, (2014) [arXiv:1407.3513v2]

[8] M. De Serio on behalf of the OPERA Collaboration, "Observation of $v_{\mu} \rightarrow v_{\tau}$ oscillations in the CNGS beam with the OPERA experiment", these Conference Proceedings

[9] V. Tioukov on behalf of the OPERA Collaboration, " $v_{\mu} \rightarrow v_{e}$ oscillation search in OPERA data", these Conference Proceedings

[10] N. Agafonova et al. [OPERA Collaboration], EPJC 74, 2986 (2014)

[11] H. Ishida et al., PTEP 9, 093 C01 (2014)

[12] A. Kayis-Topaksu et al. [CHORUS Collaboration], New. J. Phys.13, 093002 (2011) 